\title{
Erratum to: Solitary plasmacytoma: population-based analysis of survival trends and effect of various treatment modalities in the USA
}

Nishitha Thumallapally ${ }^{1 *}$, Ahmed Meshref ${ }^{2}$, Mohammed Mousa² and Terenig Terjanian ${ }^{3}$

\section{Erratum}

After publication of the original article [1] the authors found the following errors had occurred:

1. Figure titles were incorrect:

- Figure 1 should be titled: Kaplan-Meier survival curve comparing sequence of RT in patients who received RT and surgery (Fig. 1)

- Figure 2 should be titled: Kaplan-Meier survival curve comparing patients who received RT vs. no RT (Fig. 2)

- Figure 3 should be titled: Kaplan-Meier survival curve comparing patients who received surgery vs. those that did not (Fig. 3)

2. In Table 6 , all $P$ values should be $<0.001$ instead of $>0.001$ (Table 6)

Corrected versions of these figures and tables are included in this Erratum.

\footnotetext{
* Correspondence: nishithareddy99@gmail.com

${ }^{1}$ Department of Medicine, Staten Island University Hospital, 475 Seaview

Avenue, Staten Island, NY 10305, USA
} 


\section{Corrected Figure 1}

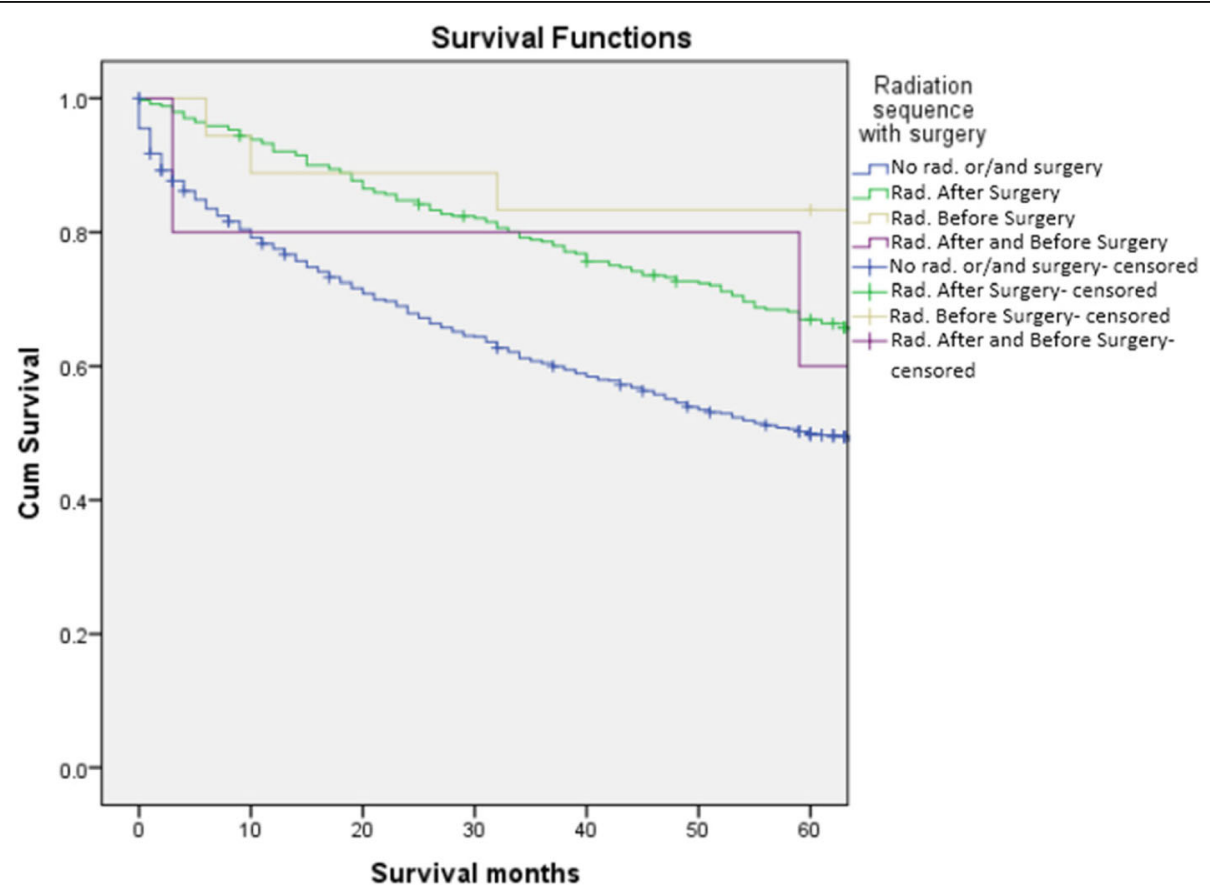

Fig. 1 Kaplan-Meier survival curve comparing sequence of RT in patients who received RT and surgery

Corrected Figure 2

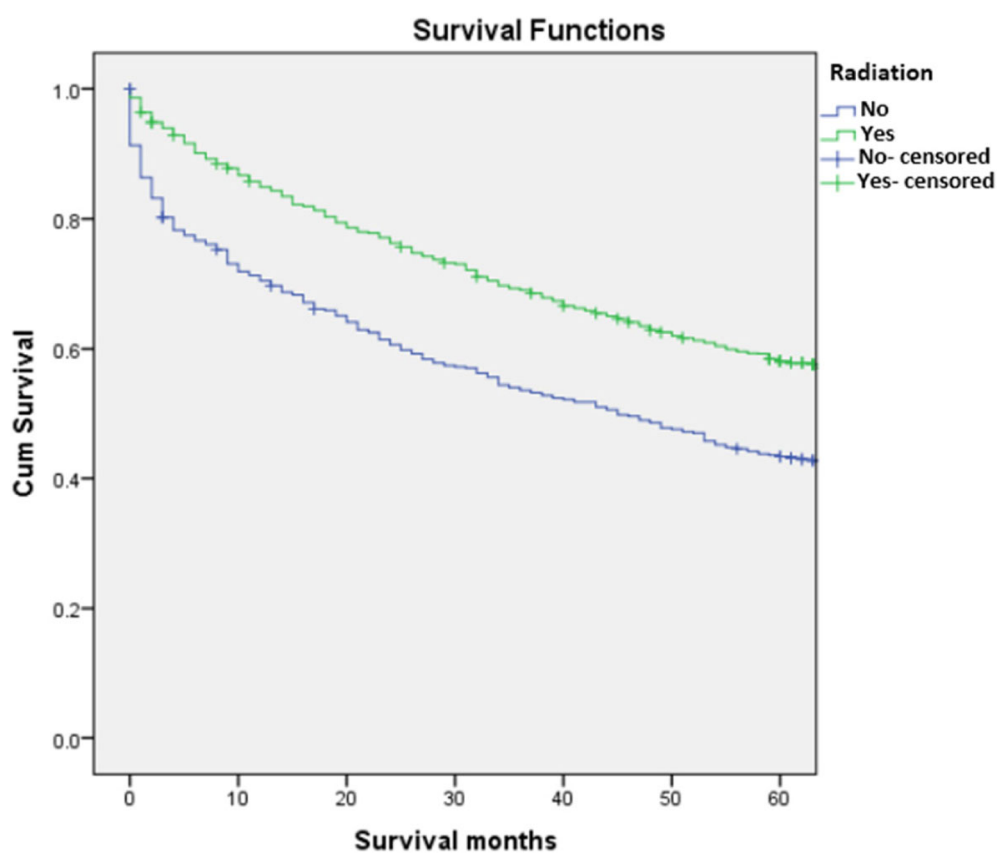

Fig. 2 Kaplan-Meier survival curve comparing patients who received RT vs. no RT 


\section{Corrected Figure 3}

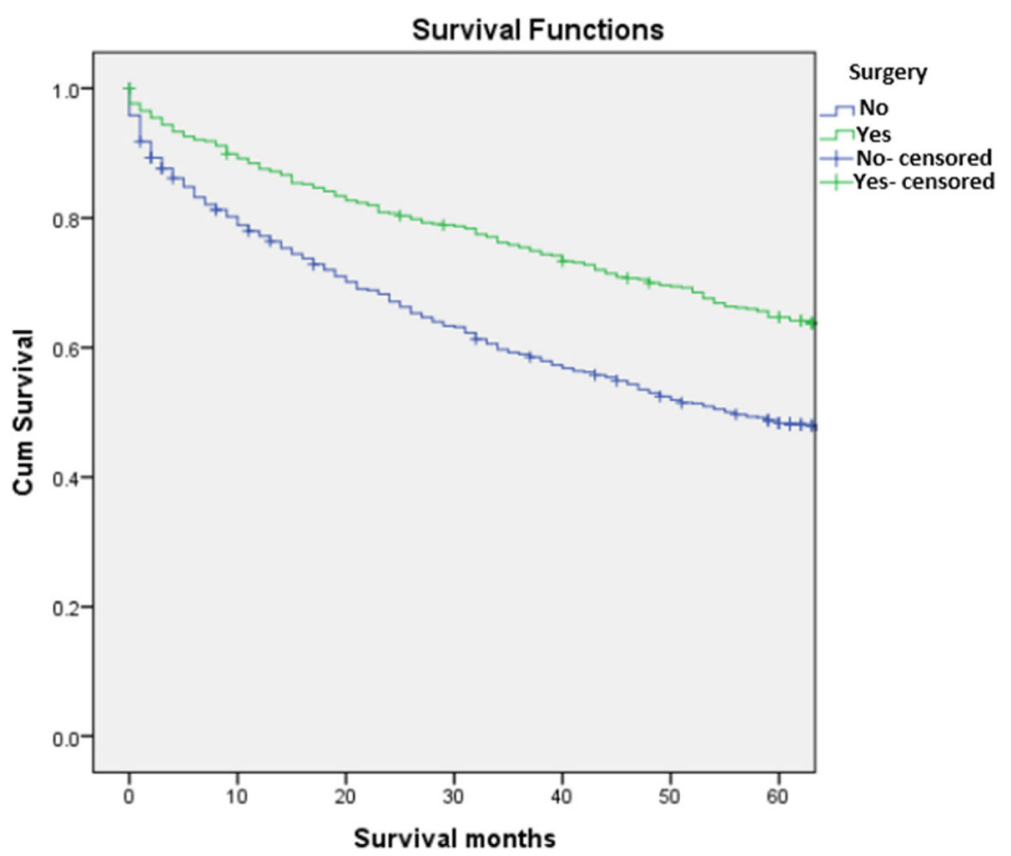

Fig. 3 Kaplan-Meier survival curve comparing patients who received surgery vs. those that did not

\section{Corrected Table 6}

Table 6 Cox proportional hazards model for prognostic factors

\begin{tabular}{lllll}
\hline Variables & $\begin{array}{l}\text { Hazard } \\
\text { ratio }\end{array}$ & \multicolumn{2}{l}{$95 \% \mathrm{Cl}$} & $P$ value \\
& & Lower & Upper & \\
\hline Age $<60$ years & 0.380 & 0.330 & 0.440 & $<0.001$ \\
$\begin{array}{l}\text { Radiation sequence } \\
\text { with surgery }\end{array}$ & 1.226 & .966 & 1.557 & .094 \\
$\begin{array}{l}\text { Radiation } \\
\text { Surgery }\end{array}$ & 0.597 & 0.522 & 0.684 & $<0.001$ \\
\hline
\end{tabular}

\section{Author details}

${ }^{1}$ Department of Medicine, Staten Island University Hospital, 475 Seaview Avenue, Staten Island, NY 10305, USA. ²Department of Medicine, Suez Canal University, Ismailia, Egypt. ${ }^{3}$ Department of Hematology/oncology, Staten Island University Hospital, Staten Island, NY, USA.

Received: 13 June 2017 Accepted: 13 June 2017

Published online: 23 June 2017

\section{Reference}

1. Thumallapally, N, Meshref, A, Mousa, M and Terjanian, T. Solitary plasmacytoma: population-based analysis of survival trends and effect of various treatment modalities in the USA, BMC Cancer, 2017, 17:13, doi:10.1186/s12885-016-3015-5 